\title{
Diagnostic Accuracy of Hexaminolevulinate in a Cohort of Patients Undergoing Radical Cystectomy
}

\author{
Vincenzo Pagliarulo, MD, Stefano Alba, MD,2 Maria Filomena Gallone, MD, \\ Savino Di Stasi, MD, Luigi Cormio, MD, Tommasangelo Petitti, MD, Maurizio Buscarini, MD? \\ Paolo Minafra, MD, and Giuseppe Carrieri, $\mathrm{MD}^{5}$
}

\begin{abstract}
Purpose: To compare the accuracy of white light cystoscopy (WLC) and blue light cystoscopy (BLC) in a cohort of patients undergoing radical cystectomy (RC) for previously resected urothelial bladder cancer.

Patients and Methods: A cohort of patients undergoing RC received WLC and BLC prior radical surgery. To evaluate the residual tumor rate, the bladder was inspected after its removal and normal appearing mucosa sampled for histologic analysis. Lesions detected under WLC, BLC, or both, and biopsy samples from normal appearing mucosa, were all recorded separately.

Results: Starting 2011, 64 patients underwent WLC and BLC prior cystectomy. Overall, 540 tissue samples were collected during cystoscopy and from normal appearing mucosa. Residual disease was found in 31/64 (48.4\%) patients, including 27 (42.1\%) cases of carcinoma in situ (CIS). The accuracy of BLC was much higher than WLC, both in the diagnosis of any residual disease $(87.1 \%$ vs $32.3 \%$, and $87.9 \%$ vs $51.5 \%$, for sensitivity and specificity, respectively), as of CIS only (92.6\% vs $29.6 \%$ and $83.8 \%$ vs $51.4 \%$ ). We further evaluated the diagnostic accuracy as a result of the analysis on all specimens collected during the study. A total of 535 specimens were analyzed, and 58 specimens with residual disease were found, including 48 CIS foci. Again, detection rates and measures of accuracy were much better for BLC vs WLC, both overall $(86.2 \% v s 31 \%$, and 98.3\% vs $93.3 \%$, for sensitivity and specificity, respectively), and when CIS only was considered (89.6\% vs $31.2 \%$ and $96.9 \%$ vs $92.8 \%)$.

Conclusions: Although BLC missed $12.9 \%$ of positive patients, and $7.4 \%$ of those with CIS, the agreement between BLC diagnostic accuracy and the definitive pathology was very robust.
\end{abstract}

Keywords: Bladder cancer, carcinoma in situ, photodynamic diagnosis, hexaminolevulinate

\section{Introduction}

$\mathbf{P}$ Hotodynamic Diagnosis (PDD) is based on fluorescence emission by cancer tissue. For reasons that are not completely understood, photosensitizers supplied exogenously increase the concentration of protoporphyrin IX (PpIX) preferentially in cancer cells. As a porphyrin, PpIX fluorescence may be excited resulting in a typical red emission in the $625-725 \mathrm{~nm}$ region, which has been exploited to increase the accuracy of several diagnostic procedures. ${ }^{1}$ In- travesical administration of 5-aminolevulinic acid or its derivatives has been used for the photodiagnosis of bladder tumors and for fluorescence-guided transurethral resection (TUR). Several meta-analyses found that blue light cystoscopy (BLC) with hexaminolevulinate detects significantly more Ta, T1, and, importantly, more carcinoma in situ (CIS) as compared to standard white light cystoscopy (WLC). ${ }^{2-5}$ Indeed, CIS is a challenging entity for urologists, being a flat urothelial lesion difficult to detect during routine cystoscopy. Conventionally, CIS is suspected in patients with positive

\footnotetext{
${ }^{1}$ Urology and Andrology Unit, Department of Emergency and Organ Transplantation, University Aldo Moro, Bari, Italy.

${ }^{2}$ Department of Urology and Mini-Invasive Surgery, Romolo Hospital, Crotone, Italy.

${ }^{3}$ Department of Biomedical Sciences and Human Oncology, University Aldo Moro, Bari, Italy.

${ }_{5}^{4}$ Department of Surgery/Urology, Tor Vergata University, Rome, Italy.

${ }^{5}$ Department of Urology and Renal Transplantation, University of Foggia, Foggia, Italy.

${ }^{6}$ Department of Epidemiology, University Hospital Campus Bio-Medico, Rome, Italy.

${ }^{7}$ Department of Urology, University Hospital Campus Bio-Medico, Rome, Italy.
} 
urinary cytology, requiring further diagnostic evaluation including upper urinary tract imaging and collection of coldcup biopsies from suspicious and normal looking mucosa of the bladder. However, this approach is unreliable, as it provides a sensitivity of only $55 \%$ for the detection of bladder CIS. ${ }^{6}$

Therefore, when CIS is suspected, contemporary EAU guidelines suggest to practice fluorescence-guided biopsies in place of traditionally performed multiple random biopsies. ${ }^{7}$ Nonetheless, several concerns remain regarding the possibility of diagnoses missed by BLC, as no study has properly investigated the bladder mucosa appearing normal during BLC and examined the false negative rate of PDD in the detection of urothelial bladder cancer (UBC).

The current study was conducted on a population of patients undergoing radical cystectomy (RC) for UBC, with the goal to compare the accuracy of WLC and BLC in detecting residual disease and CIS far from a previously resected tumor.

\section{Patients and Methods}

\section{Study design}

Since 2008 the use of hexaminolevulinate was introduced in our department as a routine practice for selected cases of nonmuscle invasive bladder cancer (NMIBC). Between October 2011 and September 2015, a single-center, prospective study was performed to assess the diagnostic accuracy of BLC in a cohort of patients undergoing RC. The study was designed as a within-patient evaluation. Immediately before $\mathrm{RC}$, eligible patients received a WLC and BLC as detailed later on. To evaluate the residual tumor rate, after removal, the bladder was inspected and normal appearing mucosa sampled for histologic analysis. All patients undergoing a $\mathrm{RC}$ were potential candidates. Exclusion criteria included an incompletely resected bladder cancer at the TUR preceding cystectomy, multifocal disease (more than three lesions), gross hematuria, non urothelial cancers, and sensitivity to levulinate. Patients undergoing a neoadjuvant chemotherapy protocol were also excluded. Subjects previously treated with endovesical bacillus Calmette-Guerin or endovesical chemotherapy were eligible.
All procedures described in the study were performed in accordance with the ethical standards of the Institutional Research Committee and with the 1964 Helsinki declaration and its later amendments. All patients provided written informed consent and the study was approved by the University of Bari Institutional Review Board (proposal No. 3595/ 2010).

\section{Hexaminolevulinate treatment and endoscopy}

Hexaminolevulinate (Hexvix ${ }^{\circledR}$; Ipsen, France) was supplied as $85 \mathrm{mg}$ powder for reconstitution in $50 \mathrm{~mL}$ phosphatebuffered saline. The solution was instilled into the bladder and retained for 1 hour. Bladders were totally voided from any residual solution before starting cystoscopy. A dedicated system was used for WLC and BLC. Specifically, a xenon D-Light C source ( $300 \mathrm{~W}$ ) (Storz GmbH, Germany), delivering excitation at a wavelength between 380 and $440 \mathrm{~nm}$, was integrated to specific PDD fiberoptic light cords and telescopes $\left(30^{\circ}\right.$ lens $)$.

\section{Endoscopy and biopsy sampling}

This procedure consisted of three main steps. (A) Careful inspection of bladder mucosa, first under white light, and then under fluorescence mode. All white light and fluorescing lesions were documented and compared. Attention was paid to whether tumors detected in the initial WLC showed fluorescence and vice versa. A documentation form was used to record the location of previously resected tumor and number and location of other suspicious areas. (B) Cold cup biopsies of all suspicious lesions were taken. Each specimen was kept in a separate container labeled as positive during WLC, BLC, or both. Whenever endoscopy, either WLE or BLE, resulted negative, no biopsies were performed at this time. The areas of previous TUR were not sampled during cystoscopy. All endoscopic procedures were performed by the same urologist (V.P.). (C) After removal, the bladder was inspected by a urologist as previously described. ${ }^{8}$ Briefly, the bladder wall was opened from the urethra to the bladder dome using scissors and the bladder surface examined. All sites previously biopsied (including ulcerated, exophytic/ nodular, or more subtle mucosal alterations) were identified.
FIG. 1. Example of bladder sampling.

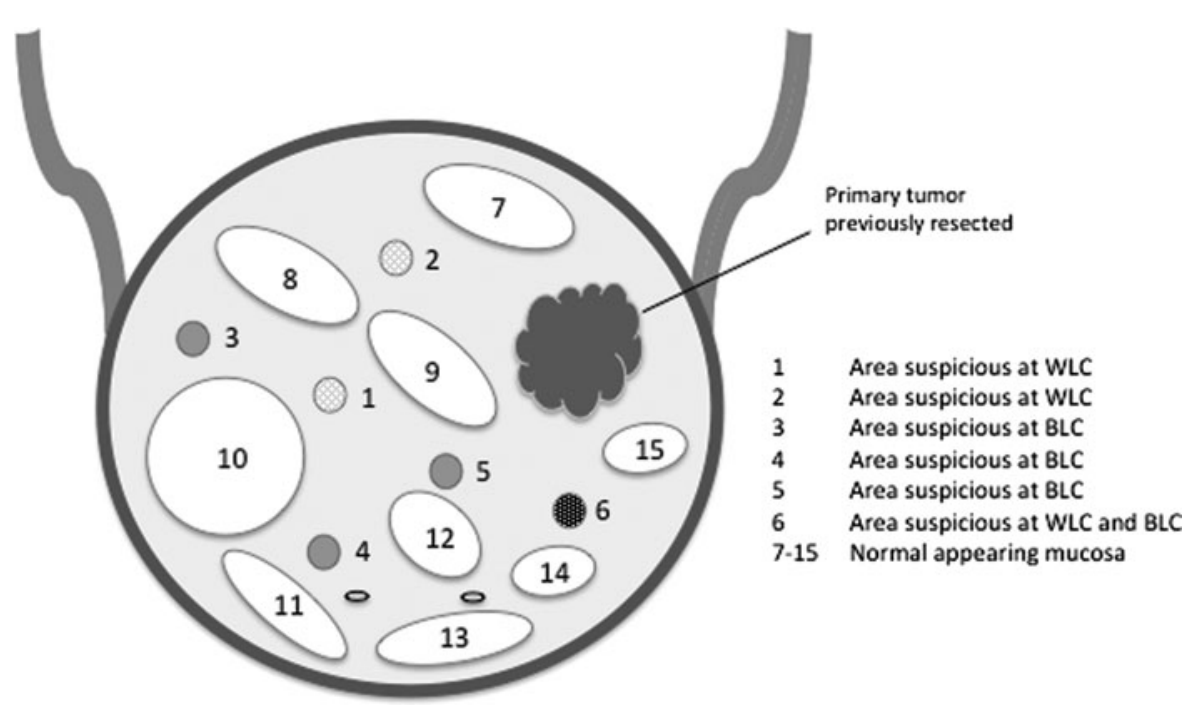


Extensive sampling of normal appearing mucosa was performed using scissors. Per each patient, all biopsy samples of normal mucosa were kept in a common container, formalin fixed, and sent to the pathologist along with the previously described samples. Figure 1 is an example of how the bladder was sampled during cystoscopy and after cystectomy.

\section{Histologic examination}

Samples were described according to their histologic appearance, that is normal urothelium, cystitis (follicular or granulomatous), flat urothelial hyperplasia, low-grade intraurothelial neoplasia (mild or moderate dysplasia), high-grade intra-urothelial neoplasia (high-grade dysplasia and CIS), and papillary urothelial neoplasia. Each sample was staged according to the 2002 UICC/American Joint Committee on Cancer system ${ }^{9}$ and graded. ${ }^{10}$

\section{Database and statistical analysis}

For each patient, continuous variables were reported as means, with standard deviation and range. Categorical variables were reported as absolute frequency and percentages. We performed two different analyses of accuracy, which separately considered patients and collected samples. In the "per patient" analysis, sensitivity, specificity, and positive (PPV) and negative (NPV) predictive values for each technique (WLC or BLC) were calculated comparing the results of endoscopy (positive or negative) with the histologic results of all biopsy samples and bladder stripping combined. Specifically, we scored the following as true positive: patient with a histology of high-grade dysplasia, CIS, and papillary urothelial neoplasia on at least one specimen collected after a positive endoscopy (WLC or BLC). False positive: patient with a histology of cystitis, hyperplasia, or mild/moderate dysplasia on all specimens collected after a positive endoscopy (WLC or BLC). True negative: patient with a negative endoscopy (WLC or BLC) and negative histology from any collected specimen. False negative: patient with a negative endoscopy (WLC or BLC) and a histological diagnosis of high-grade dysplasia, CIS, or papillary urothelial neoplasia from any collected specimen. As all specimens collected during cystoscopy were kept and analyzed separately by the pathologist, we also provided a "per specimen" analysis, in which detection rate and diagnostic accuracy were evaluated as a result of individual analysis on all the specimens collected in the entire cohort. For the "per specimen" analysis the gold standard for endoscopy was the histological diagnosis of each single specimen. For each value of sensitivity, specificity, PPV, and NPV the $95 \%$ confidence interval was indicated. The Cohen $\mathrm{k}$ test was used to evaluate the agreement between each endoscopic techniques and the final pathology classification of patient and specimens. ${ }^{11}$ For this test a $p$-value $<0.05$ was considered as significant. All analyses were performed using STATA MP12 software.

\section{Results}

\section{Patient characteristics}

Between October 2011 and September 2015, 126 patients underwent RC for UBC at our institution and were
Table 1. Patient Characteristics

\begin{tabular}{|c|c|}
\hline & Values \\
\hline Number of eligible patients & 64 \\
\hline Age (years), mean \pm SD (range) & $69.1 \pm 8.8(37-86)$ \\
\hline Male gender, $n(\%)$ & $58(90.6)$ \\
\hline $\begin{array}{l}\text { History of BCG treatment } \\
\quad(n \text { patients }), n(\%)\end{array}$ & $18(28.1)$ \\
\hline $\begin{array}{l}\text { Time between BCG treatment } \\
\text { and TUR (years), } \\
\text { mean } \pm S D \text { (range) }\end{array}$ & $1.6 \pm 2.1(0.1-6.8)$ \\
\hline $\begin{array}{l}\text { Time between BCG treatment } \\
\text { and cystectomy (years), } \\
\text { mean } \pm \text { SD (range) }\end{array}$ & $1.8 \pm 2.1(0.3-7.0)$ \\
\hline $\begin{array}{l}\text { Time between last TUR } \\
\text { and cystectomy (months), } \\
\text { mean } \pm \mathrm{SD} \text { (range) }\end{array}$ & $2.7 \pm 2.6(0.5-17.3)$ \\
\hline $\begin{array}{l}\text { Lesion diameter at TUR }(\mathrm{mm}) \\
\text { mean } \pm \text { SD (range) }\end{array}$ & $31.9 \pm 12.7(10.0-55.0)$ \\
\hline \multicolumn{2}{|l|}{ Disease focality at TUR, $n(\%)$} \\
\hline 1 & $48(75.0)$ \\
\hline 2 & $6(9.4)$ \\
\hline 3 & $10(15.6)$ \\
\hline \multicolumn{2}{|c|}{ Pathological stage and grade at TUR, $n(\%)$} \\
\hline Ta-1 & $21(32.8)$ \\
\hline$T 2$ & $31(48.4)$ \\
\hline$T x$ & $12(18.7)$ \\
\hline G3 & $64(100)$ \\
\hline Cis & $12(18.7)$ \\
\hline \multicolumn{2}{|c|}{ Pathological stage and grade at cystectomy, $n(\%)$} \\
\hline$T a-1$ & $11(17.2)$ \\
\hline$T 2$ & $19(29.6)$ \\
\hline$T 3$ & $31(48.4)$ \\
\hline T4 & $3(4.7)$ \\
\hline G3 & 64 (100) \\
\hline NO & $44(68.7)$ \\
\hline$N+$ & $14(21.9)$ \\
\hline$N x$ & $6(9.4)$ \\
\hline
\end{tabular}

$\mathrm{BCG}=$ bacillus Calmette-Guerin; TUR $=$ transurethral resection.

screened for inclusion in the present study. A total of 44 patients were excluded because of incompletely resected bladder cancer during staging TUR, multifocal disease, or because of a gross hematuria incompatible with adequate BLC; further, six patients underwent neo-adjuvant chemotherapy. Finally, 12 patients were excluded after hexaminolevulinate instillation, either because of suboptimal

\section{Table 2. Specimens Collected During Cystoscopy AND AT THE TIME OF BladDER STRIPPING After Radical Cystectomy}

\begin{tabular}{lccc}
\hline & $\begin{array}{c}\text { Collected } \\
\text { spicemens }\end{array}$ & $\begin{array}{c}\text { Not } \\
\text { eligible }\end{array}$ & $\begin{array}{c}\text { Analyzed } \\
\text { spicemens }\end{array}$ \\
\hline WLC only & 34 & 4 & 30 \\
BLC only & 38 & 0 & 38 \\
Positive both to WLC & 21 & 1 & 20 \\
$\quad$ and BLC & 447 & 0 & 447 \\
Normal appearing bladder & 540 & 5 & 535 \\
Total & & \\
\hline
\end{tabular}

$\mathrm{BLC}=$ blue light cystoscopy; $\mathrm{WLC}=$ white light cystoscopy. 
Table 3. Detection Rates, Sensitivity, Specificity, Positive and Negative Predictive Values of White and Blue Light Cystoscopy Compared Analysis on All Patients

\begin{tabular}{|c|c|c|c|c|}
\hline A. Detection rates & $W L C, \mathrm{n} / \mathrm{N}(\%)$ & $B L C, \mathrm{n} / \mathrm{N}(\%)$ & Stripping, n/N (\%) & Final diagnosis, $\mathrm{n} / \mathrm{N}(\%)$ \\
\hline Patients with CIS & $8 / 64(12.5)$ & 25/64 (39.0) & $4 / 64(6.3)$ & $27 / 64(42.2)$ \\
\hline Patients with lesions other than CIS & $3 / 64(4.7)$ & 7/64 (10.9) & $1 / 64(1.6)$ & 9/64 (14.1) \\
\hline Patients with any tumor & $10 / 64(15.6)$ & $27 / 64(42.2)$ & $5 / 64(7.8)$ & $31 / 64(48.4)$ \\
\hline B. Patients with CIS & \multicolumn{2}{|c|}{$W L C$} & \multicolumn{2}{|r|}{$B L C$} \\
\hline & $\mathrm{n} / \mathrm{N}$ & $\%(95 \% C I)$ & $\mathrm{n} / \mathrm{N}$ & $\%(95 \% C I)$ \\
\hline Sensitivity & $8 / 27$ & $29.6(20.4-57.8)$ & $25 / 27$ & $92.6(75.7-99.1)$ \\
\hline Specificity & $19 / 37$ & $51.4(34.4-68.1)$ & $31 / 37$ & $83.8(68.0-93.8)$ \\
\hline PPV & $17 / 35$ & $48.6(31.4-66.0)$ & $25 / 31$ & $80.6(62.5-92.5)$ \\
\hline NPV & $19 / 29$ & $65.5(45.7-82.1)$ & $31 / 33$ & $93.9(79.8-99.3)$ \\
\hline C. Patients with any tumor & \multicolumn{2}{|c|}{$W L C$} & \multicolumn{2}{|r|}{$B L C$} \\
\hline & $\mathrm{n} / \mathrm{N}$ & $\%(95 \% C I)$ & $\mathrm{n} / \mathrm{N}$ & $\%(95 \% C I)$ \\
\hline Sensitivity & $10 / 31$ & $32.3(16.7-51.4)$ & $27 / 31$ & $87.1(70.2-96.4)$ \\
\hline Specificity & $17 / 33$ & $51.5(33.5-69.2)$ & $29 / 33$ & $87.9(71.8-96.6)$ \\
\hline PPV & $16 / 26$ & $28.6(20.2-59.4)$ & $27 / 31$ & $87.1(70.2-96.4)$ \\
\hline NPV & $17 / 38$ & $44.7(28.6-61.7)$ & $29 / 33$ & 87.9 (71.8-96.6) \\
\hline
\end{tabular}

$\mathrm{CI}=$ confidence interval; $\mathrm{CIS}=$ carcinoma in $\mathrm{situ}$; NPV = negative predictive value; $\mathrm{PPV}=$ positive predictive value .

tissue fluorescence, or because of disease burden discordant with the previous TUR. Finally, 64 patients underwent proper cystoscopy followed by cystectomy and were analyzed in the current study (Table 1 ).

\section{Collected specimens}

Overall, 540 biopsies were collected (Table 2). Of the total, 93 were taken during cystoscopy. In 21 cases there was an agreement between the two techniques so that a suspicious area seen at WLC was also fluorescent at BLC. Five specimens from cold cup biopsies had quality issues and could not be assessed by the pathologist. Finally, 447 tissue samples were collected and analyzed as a result of bladder stripping after cystectomy.

\section{Detection rates and diagnostic accuracy}

Measures of accuracy were performed on a "per patient", (Table 3) and "per specimen" (Table 4) basis; all results are reported in the corresponding tables.

According to the final pathology on the entire cohort, 31/64 patients had residual disease far from the area of primary TUR; among those, 27/64 were recognized as CIS (including two cases of high-grade dysplasia) (Table 3 ). In terms of detection rates, BLC was superior to WLC, both in diagnosing any residual disease, as in diagnosing residual CIS (Table 3). There were only $2 / 64$ patients $(3.1 \%)$, in which neither BLC nor WLC were able to identify residual UBC (CIS in both cases). In terms of diagnostic accuracy, we found that sensitivity, specificity, PPV, and NPV were much better for BLC as

Table 4. Detection Rates, Sensitivity, Specificity, Positive and Negative Predictive Values of White and Blue Light Cystoscopy Compared Analysis on All Specimens

\begin{tabular}{|c|c|c|c|c|}
\hline A. Detection rates & $W L C, \mathrm{n} / \mathrm{N}(\%)$ & $B L C, \mathrm{n} / \mathrm{N}(\%)$ & Stripping, $\mathrm{n} / \mathrm{N}(\%)$ & Final diagnosis, $\mathrm{n} / \mathrm{N}$ \\
\hline Specimens with CIS & $15 / 46(32.6)$ & 43/46 (93.4) & $5 / 46(10.8)$ & $48 / 535$ \\
\hline Specimens with lesions other than CIS & $3 / 10(30.0)$ & $7 / 10(70.0)$ & $1 / 10(10.0)$ & $10 / 535$ \\
\hline Specimens with any tumor & $18 / 58(31.0)$ & $50 / 58(86.2)$ & $6 / 58(10.3)$ & $58 / 535$ \\
\hline B. Specimens with CIS & \multicolumn{2}{|c|}{$W L C$} & \multicolumn{2}{|c|}{$B L C$} \\
\hline & $\mathrm{n} / \mathrm{N}$ & $\%(95 \% C I)$ & $\mathrm{n} / \mathrm{N}$ & $\%(95 \% C I)$ \\
\hline Sensitivity & $15 / 48$ & $31.2(18.7-46.3)$ & $43 / 48$ & $89.6(77.3-96.5)$ \\
\hline Specificity & $454 / 487$ & $92.8(90.1-94.9)$ & $472 / 487$ & $96.9(95.0-98.3)$ \\
\hline PPV & $15 / 50$ & $30.0(17.9-44.6)$ & $43 / 58$ & $74.1(61.0-84.7)$ \\
\hline NPV & $454 / 485$ & $93.2(90.6-95.3)$ & $472 / 477$ & $99.0(97.6-99.7)$ \\
\hline C. Specimens with any tumor & \multicolumn{2}{|c|}{$W L C$} & \multicolumn{2}{|c|}{$B L C$} \\
\hline & $\mathrm{n} / \mathrm{N}$ & $\%(95 \% C I)$ & $\mathrm{n} / \mathrm{N}$ & $\%(95 \% C I)$ \\
\hline Sensitivity & $18 / 58$ & $31.0(19.5-44.5)$ & $50 / 58$ & $86.2(74.6-93.9)$ \\
\hline Specificity & $445 / 477$ & $93.3(90.7-95.4)$ & $469 / 477$ & $98.3(96.7-99.3)$ \\
\hline PPV & $18 / 50$ & $36.0(22.9-50.8)$ & $50 / 58$ & $86.2(74.6-93.9)$ \\
\hline NPV & $445 / 485$ & 91.8 (88.9-94.0) & $469 / 477$ & 98.3 (96.7-99.3 \\
\hline
\end{tabular}


Table 5. Number of Lesions Found After White and Blue Light Cystoscopy, and Overall

\begin{tabular}{lccc}
\hline & $W L C, \mathrm{n}(\%)$ & $B L C, \mathrm{n}(\%)$ & Overall, $\mathrm{n}(\%)$ \\
\hline 1 lesion & $4(40.0)$ & $11(40.75)$ & $13(41.9)$ \\
2 lesions & $4(40.0)$ & $11(40.75)$ & $11(35.5)$ \\
3 lesions & $2(20.0)$ & $3(11.1)$ & $5(16.1)$ \\
4 lesions & $0(0.0)$ & $2(7.4)$ & $2(6.5)$ \\
Total & $10(100.0)$ & $27(100.0)$ & $31(100.00)$ \\
\hline
\end{tabular}

compared to WLC, both in diagnosing patients with residual CIS, as those with any residual disease (Table 3 ).

Among all specimens collected during endoscopy and bladder stripping, a total of 58/535 resulted positive at the final pathology report, including 48/535 specimens with CIS (Table 4). Only 6/535 specimens with UBC, consisting of five cases of CIS and one T1G3, were completely overlooked both by BLC and WLC. In the analysis on all specimens, BLC had again superior detection rates as compared to WLC, both in diagnosing any residual disease, as in diagnosing residual CIS (Table 4). Further, in terms of diagnostic accuracy, BLC performed consistently better than WLC, by all means (Table 4). When using Cohen's kappa coefficient to further compare cystoscopy to final pathology, BLC resulted in a much higher agreement compared to WLC, both in terms of diagnosis of positive patients $(0.75 \mathrm{vs}$ $0.13)$, as diagnosis of any positive lesion (0.85 vs 0.26$)$.

\section{Number of lesions}

Of the 31 patients found positive at definitive pathology, 13 had a single lesion, while 18 had multifocal disease (Table 5). During cystoscopy, uni and multifocal disease were discovered in 11 and 16 patients with BLC, and in 4 and 6 patients using WLC, respectively. As expected, BLC exhibited an almost perfect agreement with final diagnosis compared to WLC (kappa: 0.89 vs 0.27).

\section{Discussion}

Urinary tract CIS reflects a challenging diagnostic entity for urologists. The combination of urinary cytology and cystoscopy with multiple bladder biopsies (MBB) has been the reference standard for CIS detection and surveillance. However, a study specifically designed to address the diagnostic accuracy of MBB revealed a sensitivity of only $55 \%$. $^{6}$ The limitations of cytology and cystoscopy with MBB became more evident in studies comparing standard WLC with BLC. These studies have been reviewed by Rink and colleagues, who reported that, when WLC is used, the overall rates of undiagnosed CIS range from $16 \%$ to $76 \% .^{12}$ These findings are further supported by four meta-analyses demonstrating that PDD significantly improves the detection of CIS by $23 \%$ to $40 \% .^{2-5}$

One limitation of all studies evaluating the diagnostic accuracy of BLC is that none of them was specifically designed to explore the normal appearing mucosa in patients with NMIBC. Specifically, in a study by Colombo, eight random biopsies were performed whenever no suspicious areas could be seen in normal appearing urothelium ${ }^{13}$; in other cases, normal appearing mucosa was checked occasionally, ${ }^{14-16}$ or as a single control biopsy. ${ }^{17-19}$ As none of the previous studies has thoroughly explored the normal appearing urothelium, little is known about the real diagnostic accuracy of fluorescent cystoscopy. This study was specifically designed to address this point. Overall, 447 bladder samples were taken after cystectomy to compare WLC and BLC to a proper reference standard. Residual disease was found in 31/64 (48.4\%) patients; among those, there were 27 (42.1\%) cases of pathologic flat lesion (25 CIS and 2 high-grade dysplasia). Importantly, only 4/31 patients with any residual disease, and only $2 / 27$ patients with a flat lesion were unrecognized at BLC. The performance of WLC was very different, as 21/31 patients with any residual disease, and 18/27 patients with a flat lesion were undiagnosed. Finally, the overall NMIBC detection accuracy showed a substantial improvement owing to the use of BLC vs WLC, both in terms of sensitivity and specificity $(87.1 \%$ vs $45.5 \%$ and $87.9 \%$ vs $40.5 \%$, respectively).

As CIS may be multifocal, per each patient we meticulously analyzed each biopsy sample separately to provide information related to the overall per biopsy detection rate. Among the 31 positive patients, a total of 58 areas of residual disease were found, including 46 CIS foci. Again, accuracy figures resulted very different between the two methods, as sensitivity and specificity rates were $86.2 \%$ and $98.3 \%$ for BLC, as compared to $31.0 \%$ and $93.3 \%$ for WLC. Importantly, these results are interesting when compared to those obtained by Gudjónsson and colleagues. In a similar cohort of patients undergoing $\mathrm{RC}$, they found that detection of CIS by bladder mapping was modest compared to analysis of all bladder mucosa (sensitivity $46 \%$, specificity $89 \%$ ), concluding that cold cup biopsies are unreliable for the diagnosis of CIS in bladder mucosa. ${ }^{6}$ According to these data, the use of bladder multiple cold cup biopsies, in addition to BLC, should be discouraged as it would unlikely improve the diagnostic accuracy of BLC alone.

The results of this study encourage the use of PDD whenever residual tumor is suspected; this is the case of a repeat TUR for high-risk NMIBC. Previous trials have already shown a substantial impact of BLC in terms of secondlook resection recurrence rates. In a study by Geavlete and colleagues, at the time second TUR, the rate of lesions overlooked (or incompletely resected) was higher in patients undergoing conventional vs PDD primary TUR. Specifically, among patients presenting with high-risk tumors (high-grade, CIS, T1), recurrence rates were $17 \%$ in the BLC group and $37 \%$ in the WLC group; when focusing on CIS only, the rates were $4.3 \%$ and $27.8 \%$, respectively. ${ }^{20}$ In an earlier study with a similar design, the tumor stage-related recurrence rates for BLC and WLC diagnosed NMIBC patients were $11.8 \%$ vs $36.0 \%$ and $0 \%$ vs $80 \%$ for pT1 and CIS cases, respectively. ${ }^{21}$ This study provides further evidence in support of the use of PDD. Specifically, in a cohort of patients with high risk NMIBC and MIBC we found that $12.9 \%$ and $67.7 \%$ of them had concomitant disease that was overlooked respectively by BLC and WLC; among patients with concomitant CIS, the rates were $7.4 \%$ and $70.3 \%$, respectively. Compared to the previous trials, we believe the design of this study may provide more realistic figures, first because of a thorough analysis of the normal appearing mucosa, as previously discussed. Further, in our study we provide a real measure of diagnostic accuracy of WLC and BLC, as whole bladder 
inspection is performed shortly after endoscopy. When such measure is performed weeks after a primary TUR, as in the above-mentioned trials, "recurrences" may in fact consist of new disease, residual tumors left behind after an incomplete resection, or lesions overlooked during first endoscopy.

We acknowledge there are limitations to this study. The cohort we investigated may not be comparable to a population of patients with high-risk NMIBC, as a large proportion of patients in our cohort underwent RC for MIBC; likewise, our findings may not reflect the figures of concomitant CIS in patients with NMIBC. This choice was dictated by the need to obtain a whole bladder specimen for thorough sampling of the bladder mucosa. Further, our study was conducted on a small cohort of 64 patients. We excluded all conditions that may have limited the ability to produce reliable results, as cases in which sampling and identification of normal mucosa after cystectomy would have been cumbersome, patients with a primary tumor incompletely resected at TUR, or consisting of more than three lesions. Finally, our study did not include any sampling of the prostatic urethra. However, proper examination of the prostatic urethra by PDD is not feasible, as there are no methods to retain photosensitizing agents into the urethral lumen for an adequate time.

\section{Conclusions}

We studied a cohort of patients undergoing RC for UBC completely resected during a previous TUR, and sought to compare the accuracy of WLC and BLC for the search of any residual disease far from the primary lesion/s. According to our results, CIS is a very common entity in patients with highrisk UBC (42.2\%), however, WLC is completely inadequate to recognize it as more than $70 \%$ of patients with CIS were overlooked. On the other side, the agreement between BLC accuracy and the definitive pathology was found to be very robust, as only $7.4 \%$ of the patients with CIS were unrecognized by BLC. Finally, we believe this study adds a strong argument in favor of PDD, as it and provides information regarding what to expect when the mucosa appears normal during conventional and fluorescence cystoscopy.

\section{Author Disclosure Statement}

No competing financial interests exist.

\section{References}

1. Miyake M, Ishii M, Kawashima K, Kodama T, Sugano K, Fujimoto K, Hirao Y. siRNA-mediated knockdown of the heme synthesis and degradation pathways: Modulation of treatment effect of 5-aminolevulinic acid-based photodynamic therapy in urothelial cancer cell lines. Photochem Photobiol 2009;85:1020-1027.

2. Burger M, Grossman HB, Droller M, et al. Photodynamic diagnosis of non-muscle-invasive bladder cancer with hexaminolevulinate cystoscopy: A meta-analysis of detection and recurrence based on raw data. Eur Urol 2013;64: 846-854.

3. Di Stasi SM, De Carlo F, Pagliarulo V, Masedu F, Verri C, Celestino F, Riedl C. Hexaminolevulinate hydrochloride in the detection of nonmuscle invasive cancer of the bladder. Ther Adv Urol 2015;7:339-350.
4. Kausch I, Sommerauer M, Montorsi F, Stenzl A, Jacqmin $\mathrm{D}$, Jichlinski $\mathrm{P}$, et al. Photodynamic diagnosis in nonmuscle-invasive bladder cancer: A systematic review and cumulative analysis of prospective studies. Eur Urol 2010; 57:595-606.

5. Mowatt G, N'Dow J, Vale L, Nabi G, Boachie C, Cook JA, Fraser C, Griffiths TR; Aberdeen Technology Assessment Review (TAR) Group. Photodynamic diagnosis of bladder cancer compared with white light cystoscopy: Systematic review and meta-analysis. Int J Technol Assess Health Care 2011;27:3-10.

6. Gudjonsson S, Blackberg M, Chebil G, et al. The value of bladder mapping and prostatic urethra biopsies for detection of carcinoma in situ (CIS). BJU Int 2012;110:E41-E45.

7. Babjuk M, Burger M, Zigeuner R, et al; European Association of Urology. EAU guidelines on non-muscleinvasive urothelial carcinoma of the bladder: Update 2013. Eur Urol 2013;64:639-653.

8. Lopez-Beltran A, Bassi P, Pavone-Macaluso M, Montironi R. Handling and pathology reporting of specimens with carcinoma of the urinary bladder, ureter, and renal pelvis. Eur Urol 2004;45:257-266.

9. Sobin LH, Wittekind CH. TNM Classification of Malignant Tumours, 6th ed. New York: Wiley-Liss, 2002.

10. Epstein JI, Amin MB, Reuter MD, Mostofi FK. The World Health Organisation/International Society of Urological Pathology consensus classification of urothelial (transitional cell) neoplasms of the urinary bladder. Bladder Consensus Conference Committee. Am J Surg Pathol 1998;22:1435-1448.

11. Cohen J. A coefficient of agreement for nominal scales. Educ Psychol Meas 1960;20:37-46.

12. Rink M, Babjuk M, Catto JW, et al. Hexyl aminolevulinateguided fluorescence cystoscopy in the diagnosis and follow-up of patients with non-muscle-invasive bladder cancer: A critical review of the current literature. Eur Urol 2013;64:624-638.

13. Colombo R, Naspro R, Bellinzoni P, et al. Photodynamic diagnosis for follow-up of carcinoma in situ of the bladder. Ther Clin Risk Manag 2007;3:1003-1007.

14. Hungerhuber E, Stepp H, Kriegmair M, et al. Seven years' experience with 5-aminolevulinic acid in detection of transitional cell carcinoma of the bladder. Urology 2007;69:260-264.

15. Karl A, Tritschler S, Stanislaus P, et al. Positive urine cytology but negative white-light cystoscopy: An indication for fluorescence cystoscopy? BJU Int 2009;103:484-487.

16. Zaak D, Hungerhuber E, Schneede P, et al. Role of 5aminolevulinic acid in the detection of urothelial premalignant lesions. Cancer 2002;95:1234-1238.

17. Fradet Y, Grossman HB, Gomella L, et al.; PC B302/01 Study Group. A comparison of hexaminolevulinate fluorescence cystoscopy and white light cystoscopy for the detection of carcinoma in situ in patients with bladder cancer: A phase III, multicenter study. J Urol 2007;178:68-73.

18. Grimbergen MC, van Swol CF, Jonges TG, Boon TA, van Moorselaar RJ. Reduced specificity of 5-ALA induced fluorescence in photodynamic diagnosis of transitional cell carcinoma after previous intravesical therapy. Eur Urol 2003;44:51-56.

19. Schmidbauer J, Witjes F, Schmeller N, Donat R, Susani M, Marberger M; Hexvix PCB301/01 Study Group. Improved detection of urothelial carcinoma in situ with hexaminolevulinate fluorescence cystoscopy. J Urol 2004;171:135-138.

20. Geavlete B, Jecu M, Multescu R, Georgescu D, Geavlete P. HAL bluelight cystoscopy in high-risk nonmuscle-invasive bladder cancer re-TURBT recurrence rates in a prospective, randomized study. Urology 2010;76:664-669. 
21. Filbeck T, Pichlmeier U, Knuechel R, Wieland WF, Roessler W. Clinically relevant improvement of recurrence-free survival with 5-aminolevulinic acid induced fluorescence diagnosis in patients with superficial bladder tumors. J Urol 2002;168:67-71.

Address correspondence to: Vincenzo Pagliarulo, MD Urology and Andrology Unit Department of Emergency and Organ Transplantation University Aldo Moro Piazza G. Cesare 11 Bari 70124 Italy

E-mail: vincenzo.pagliarulo@uniba.it; enzopagliarulo@yahoo.com

\section{Abbreviations Used}

$\mathrm{BCG}=$ bacillus Calmette-Guerin

$\mathrm{BLC}=$ blue light cystoscopy

CIS $=$ carcinoma in situ

$\mathrm{MBB}=$ multiple bladder biopsies

$\mathrm{NMIBC}=$ nonmuscle invasive bladder cancer

$\mathrm{NPV}=$ negative predictive value

$\mathrm{PDD}=$ photodynamic diagnosis

PpIX = protoporphyrin IX

$\mathrm{PPV}=$ positive predictive value

TUR $=$ transurethral resection

$\mathrm{UBC}=$ urothelial bladder cancer

$\mathrm{WLC}=$ white light cystoscopy 